Article

\title{
Acoustic Design of a Classical Concert Hall and Evaluation of its Acoustic Performance - A Case Study
}

\author{
Kwangbok Jeong, ${ }^{1}$ Taehoon Hong ${ }^{1 *}$, Sung Hoon $\mathrm{Kim}^{2}$, Jimin Kim${ }^{3}$, Sangyoub Lee ${ }^{4}$ \\ 1 Department of Architecture and Architectural Engineering, Yonsei University, Seoul, 03722, Republic of \\ Korea; kbjeong7@yonsei.ac.kr; hong7@yonsei.ac.kr \\ 2 Architectural Development Team, Kumho Engineering \& Construction, Seoul, 03185, Republic of Korea; \\ shkim9@kumhoenc.com \\ 3 Division of Construction Engineering and Management, Purdue University, West Lafayette, IN 47907 \& \\ Department of Architecture and Architectural Engineering, Yonsei University, Seoul, 03722, Republic of \\ Korea; kim2752@purdue.edu \\ 4 Department of Real Estate, Konkuk University, Seoul, 05029, Republic of Korea; sangyoub@konkuk.ac.kr \\ * Correspondence: hong7@yonsei.ac.kr; Tel.: +82-2-2123-5788
}

\begin{abstract}
This study aims at an acoustic design of the classical concert hall and evaluation of the acoustic performance. In terms of three acoustic parameters (i.e., reverberation time (RT), clarity (C80), and lateral fraction (LF)), this study performed acoustic simulation modeling and site measurement with the K Art Hall located in South Korea as a case study. First, in order to meet the acoustic performance of the K Art Hall (target RT: 1.4 1.7 seconds, target C80: $-2 \mathrm{~dB}$ or more $+2 \mathrm{~dB}$ or less, and target LF: 10 35\%), the finish materials and shape of the room as an interior acoustic design were determined. Second, the average values of the RT, C80, and LF using the acoustic simulation modeling were estimated at 1.4 second, $1.2 \sim 1.6 \mathrm{~dB}$, and $29 \%$, respectively. Third, the average values of the RT, C80, and LF through site measuring were measured at 1.5 1.64 second, $0.07 \sim 1.31 \mathrm{~dB}$, and $22.22 \sim 31.37 \%$, respectively. Thus, the results of both the acoustic simulation modeling and site measuring were analyzed so as to satisfy the target acoustic performance. The results of this study will help the decision-makers (i.e., owner, construction managers, etc.) to plan the classical concert hall in terms of the RT, C80, and LF.
\end{abstract}

Keywords: Acoustic design; Reverberation time; Clarity; Lateral fraction; Acoustic measurements; Classical concert hall

\section{Introduction}

The acoustic design and evaluation of the acoustic performance in the classical concert hall have been topics of interest for many years. This is because the flow of the sound delivered from the stage to the auditorium determines the overall acoustic quality [1-5]. In particular, the sound quality of the classical concert hall is largely affected by the architectural conditions - interior design, finish, material and so on). Thus, to plan a classical concert hall that satisfies the acoustic performance, the acoustic design and evaluation should be conducted considering the acoustic parameters (i.e., reverberation time (RT), clarity (C80), and lateral fraction (LF)) of the classical music from the early planning phase all the way through to operation phase [6].

Several previous studies have conducted an evaluation of the acoustic performance using the following two approaches: (i) acoustic simulation analysis and (ii) site measuring analysis.

First, several studies have conducted an acoustic simulation analysis through computer simulation programs in the early planning phase. Barron and Kissner [7] presented a possible acoustic design approach for multi-purpose auditoria. Two acoustic models were simulated using computer simulation programs. Through which, this study proposed an acoustic design approach 
that can reduce the secondary reverberation occurring in concert halls by the horizontal sound field. Garrido JA et al. [8] conducted an acoustic simulation for the Manuel de Falla Auditorium using Computer Aided Theater Technique (CATT)-Acoustic and CATT-TUCT. Based on this acoustic simulation and situ measurements, the main acoustic parameters within the Manuel de Falla Auditorium were predicted in this study. Navarro and Escolano [9] conducted the indoor acoustic simulation of the building using an acoustic diffusion equation model which was developed based on the propagation of sound particles. This study presented the state-of-art of diffusion equation model by applying several acoustic scenarios.

Second, several studies have conducted a site measuring analysis in the operation phase. Martins et al. [10] conducted an experimental comparison analysis of the acoustic performance between the timber and timber-concrete floors. The method of applying timber-concrete to a building was analyzed to satisfy both the acoustic comfort level and structural improvement. De Sant'Ana and Zannin [11] conducted an acoustic evaluation of a contemporary church based on in situ measurements. The measurement results were compared with three international standards (i.e., NBR 12179 Brazilian National Standard, ISO 3382-1 international standard, and IEC 60268-16 Standard). Arets and Orlowski [12] analyzed the sound strength and RT for six small concert halls using the site measurement. To avoid excessive loudness and obtain sufficient reverberation, the two acoustic parameters (i.e., sound strength and RT) for the small concert halls must create a balance with one another. This is because these two acoustic parameters were analyzed to closely affect one another. Mak and Wang [13] presented an overview of the prediction method in building acoustics. In this study, several acoustic prediction models (i.e., numerical model, experimental model, and analytical model) were reviewed to provide an indication of the advanced model for air-borne sound, structure-borne sound, and duct-borne sound.

Through a literature review of the acoustic performance (i.e., acoustic simulation analysis and site measuring analysis), the importance of acoustic design and evaluation of the classical concert hall from the early planning phase to operation phase was verified. However, most previous studies only performed one of the acoustic simulation analysis or site measuring analysis. Therefore, this study intends to conduct an acoustic design of the classical concert hall and its evaluation of the acoustic performance of the K Art Hall.

\section{Establishment of the interior acoustic design of the classical concert hall}

This study aimed to conduct a case study on the acoustic design and evaluation of its acoustic performance of the K Art Hall located in Seoul, South Korea. The K Art Hall is a classical concert hall completed in 2016, and the interior acoustic design of the ' $K$ ' Art Hall was conducted in the following two phases: (i) determination of the shape of room in the classical concert hall and (ii) determination of the finish materials in the classical concert hall.

\subsection{Determination of the shape of the room in the classical concert hall}

The shape of the room, which is determined by the purpose and size of the room, as well as other features, in the classical concert hall is an element that determines the sound quality. The purpose of the room is to perform classical music, and thus, the K Art Hall was designed as a 'Shoe-Box' type (refer to Figure 1). The 'Shoe-box' type is generally used in concert halls, as it provides reverberations suitable for a concert hall due to the reflection of sound based on the parallel sidewalls [14-15]. In contrast, if formed as a narrow and long 'Shoe-box' type, the refraction sound of the sidewall may be poor due to the problem of the longer distance between the stage and the back seats [16]. However, as shown in Table 1, the K Art Hall was designed to avoid the problem of poor refraction sound of the sidewall by being a medium-sized classic concert hall (e.g., building area: 1,500m2, and volume of hall: $3,500 \mathrm{~m} 3$, maximum room height: $8.0 \mathrm{~m}$, Average room length: $23 \mathrm{~m}$, etc.). 


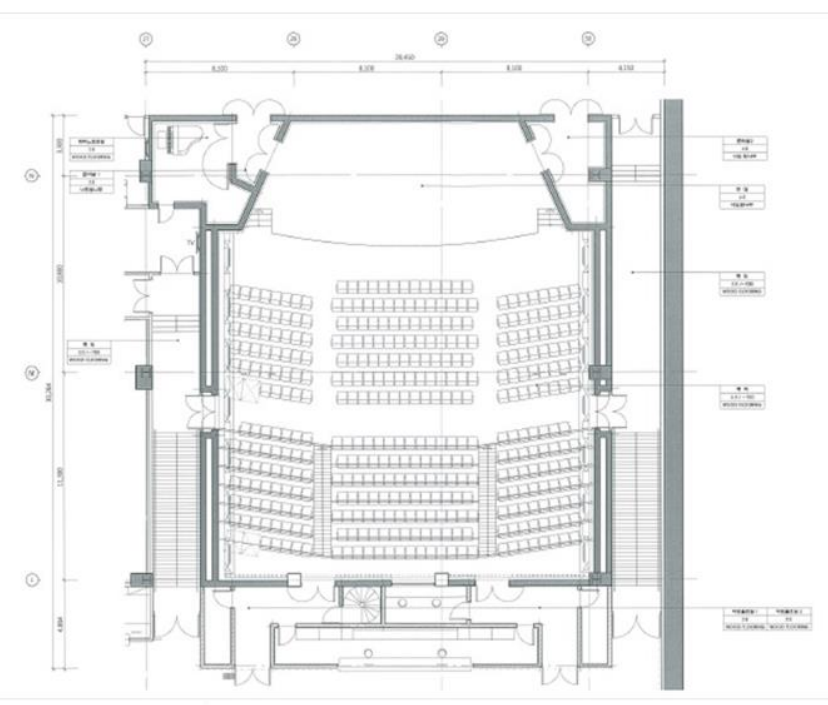

(a) Plan

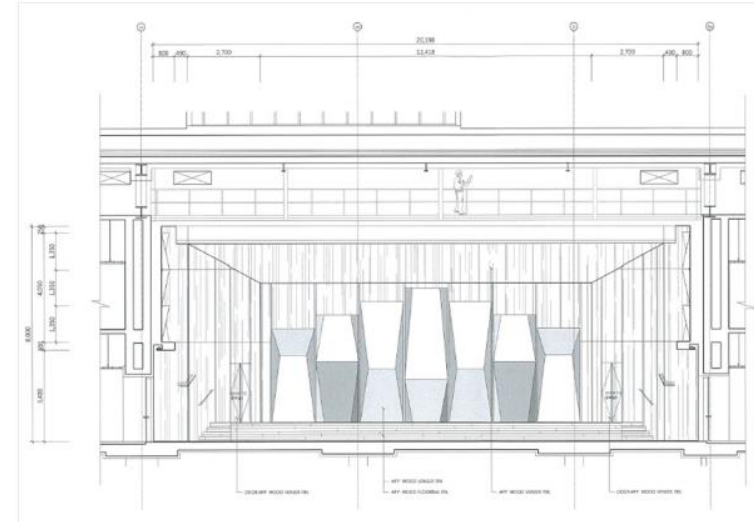

(B) Elevation - 1 (Front wall)

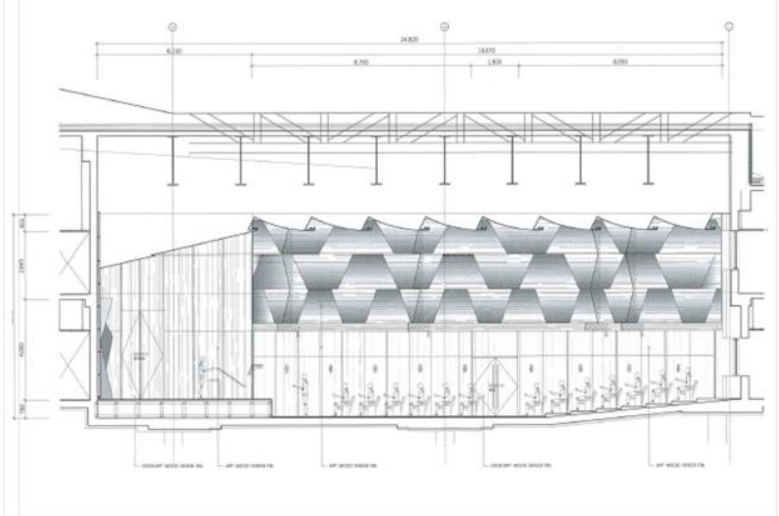

(C) Elevation - 2 (Sidewall)

Figure 1. The plan and elevation drawings of the K Art Hall

Table 1. Architectural scheme of the K Art Hall

\begin{tabular}{ccc}
\hline Class & Unit & Value \\
\hline Type of building & - & Classical concert hall \\
Building area & $\left(\mathrm{m}^{2}\right)$ & 1,500 \\
Volume of concert hall & $\left(\mathrm{m}^{3}\right)$ & 3,500 \\
Number of seats in concert hall & $(\mathrm{seats})$ & 390 \\
Volume per seat & $\left(\mathrm{m}^{3} / \mathrm{seat}\right)$ & 9.08 \\
Maximum room height & $(\mathrm{m})$ & 8.0 \\
Average room length & $(\mathrm{m})$ & 23.0 \\
Average room width & $(\mathrm{m})$ & 19.0 \\
\hline
\end{tabular}

\subsection{Determination of the finish materials in the classical concert hall}

The finish materials of a classical concert hall have a considerable effect on the hall's acoustic performance through sound reflection and diffusion [17-19]. To achieve the optimal acoustic performance of $\mathrm{K}$ Art Hall, this study planned the finish materials by considering the following two interior design factors: (i) stage and (ii) auditorium (refer to Table 2). First, the stage of the classical concert hall was designed in such a way that the sound emitted on it would be delivered to the players on the stage and the audiences in the auditorium. Considering the sound reflection and diffusion of K Art Hall, the sidewall of the stage was designed to have a trapezoidal shape, and the angle of the stage's ceiling was set to $10^{\circ}$. Also, the finish material that was chosen for the floor of the stage was 
wood, and the finish material that was chosen for the other parts (i.e., posterior wall, sound diffusers, and ceiling) was wood veneer sheet.

Second, the auditorium of the classical concert hall aims to block the flutter echoes and to deliver equational acoustic energy to the entire audience by controlling the reflection and diffusion of the sound delivered to the stage [20]. For this, sound diffusers were installed in the sidewall of the auditorium to prevent the generation of flutter echoes reflected from the parallel sidewalls. In addition, to prevent the emergence of long-path echoes caused by the sounds generated from the stage that were then reflected from the posterior wall of the auditorium to the stage, a sound absorption material was used for the posterior wall of the auditorium [21], resulting in the dissipation of the acoustic energy delivered to the posterior wall of the auditorium. The ceiling of the auditorium was designed as a closed ceiling in principle so that the equational acoustic energy can be delivered to the entire audience in the auditorium of the classical concert hall [22]. Sight glass, auditorium chairs, and soundproof doors covered with artificial leather were also used.

Meanwhile, Table 2 shows the sound absorption coefficient by frequency band for the finish materials in $\mathrm{K}$ Art Hall. The sound absorption coefficient by frequency band was shown to be between 0.06 and 0.84 , depending on the finish material. As discussed in section 3 (acoustic modeling of the classical concert hall using an acoustic simulation program), to perform acoustic modeling, these sound absorption coefficients by frequency band were used.

Table 2. Sound absorption coefficient by frequency band for the finish materials used for K Art Hall

\begin{tabular}{|c|c|c|c|c|c|c|c|}
\hline & \multirow{2}{*}{ Class } & \multirow{3}{*}{ Finish materials } & \multicolumn{5}{|c|}{$\begin{array}{c}\text { Sound absorbing coefficient by } \\
\text { frequency band }(\alpha)\end{array}$} \\
\hline & & & 125 & 250 & 500 & 1,000 & 2,000 \\
\hline \multirow{5}{*}{ Stage } & & & & & & & \\
\hline & Floor & Wood flooring & 0.15 & 0.11 & 0.1 & 0.07 & 0.06 \\
\hline & Posterior wall & Wood veneer sheet & 0.1 & 0.07 & 0.05 & 0.05 & 0.04 \\
\hline & Sound diffusers & Wood veneer sheet & 0.1 & 0.07 & 0.05 & 0.05 & 0.04 \\
\hline & Ceiling & Wood veneer sheet & 0.11 & 0.11 & 0.05 & 0.06 & 0.04 \\
\hline \multirow{5}{*}{ Auditorium } & Floor & Wood flooring & 0.04 & 0.04 & 0.07 & 0.07 & 0.06 \\
\hline & Sidewall & Wood veneer sheet & 0.1 & 0.07 & 0.05 & 0.05 & 0.04 \\
\hline & Sound diffusers on the sidewall & Wood veneer sheet & 0.1 & 0.07 & 0.05 & 0.05 & 0.04 \\
\hline & Louver on the posterior wall & Wood veneer sheet & 0.1 & 0.07 & 0.05 & 0.05 & 0.04 \\
\hline & Ceiling & Wood veneer sheet & 0.11 & 0.11 & 0.05 & 0.06 & 0.04 \\
\hline \multirow{3}{*}{ Others } & Glass & Sight glass & 0.15 & 0.05 & 0.04 & 0.03 & 0.02 \\
\hline & Chair & Auditorium chair & 0.43 & 0.62 & 0.82 & 0.78 & 0.84 \\
\hline & Soundproof door & $\begin{array}{l}\text { Soundproof door covered with } \\
\text { artificial leather }\end{array}$ & 0.06 & 0.13 & 0.1 & 0.1 & 0.1 \\
\hline
\end{tabular}

\section{Acoustic simulation modeling of the classical concert hall using an acoustic simulation program}

\subsection{Establishment of the target acoustic performance}

For the evaluation of the acoustic performance of $\mathrm{K}$ Art Hall, three acoustic parameters were established in this study: RT, C80, and LF.

First, reverberation is a phenomenon in which when a sound from an indoor sound source stops, it does not disappear immediately but dissipates gradually until it disappears. The RT is a quantitative value of the reverberation, referring to the time it takes for the average indoor sound energy density to drop by $60 \mathrm{~dB}$ after a sound of a certain density is generated and reaches the equilibrium [23]. According to Barron M. [24] and Egan M.D. [25], the recommended RT for music venues (e.g., chamber hall, recital hall, etc.) is between 1.4 and 1.7 seconds. Kim [26] proposed that the optimal RT for a music venue based on its volume and building type should be within 1.4 seconds. Thus, the target RT of K Art Hall was set as $1.4 \sim 1.7$ seconds.

Second, C80, a valuable room acoustic parameter for music, is the ratio of the sound energy before $80 \mathrm{~ms}$ (early sound energy) and after $80 \mathrm{~ms}$ (late sound energy) after the sound is generated 
from the sound source (refer to equation (1)) $[27,28]$. The greater the increase in the C80 value is, the shorter the sound connection becomes. While the clarity improves, the sound is not naturally connected. On the other hand, the greater the reduction in the C 80 value is, the longer the sound connection becomes. As the latter sound energy is delivered before the former sound energy is reduced, the two sounds overlap. According to Barron [24,29,30], the recommended C80 of a music venue is between -2 and +2 . Thus, the target $\mathrm{C} 80$ of $\mathrm{K}$ Art Hall was set to $[-2,+2]$.

$$
C_{80}=10 \log \left(\frac{E S E}{L S E}\right)
$$

where, $C_{80}$ is the clarity; ESE is the early sound energy; and LSE is the late sound energy.

Third, the LF refers to the ratio of the sound energy reflected laterally to the sound energy reflected from all directions, and can be calculated using equation (2) [31]. In other words, LF shows the sense of spatiality surrounded by sounds. According to Barron [29] and Fasold [32], and Sant'Ana and Zannin [11], the recommended LF for music venues is between 10 and $35 \%$. Thus, the target LF of K Art Hall was set to $10 \sim 35 \%$.

$L F=\frac{\int_{5 m s}^{80 m s} I R_{f 8}^{2}(t) d t}{\int_{0}^{80 m s} I R_{o}^{2}(t) d t}$

where, $L F$ is the lateral fraction; $h_{f 8}(t)$ is the room impulse response measured using a figure- 8 microphone; and $h_{S R}(t)$ is the room impulse response measured with an omnidirectional microphone.

\subsection{Analysis of the acoustic performance through acoustic simulation modeling}

In terms of the acoustic performance based on three acoustic parameters (i.e., RT, C80, and LF), the feasibility of the acoustic design of $\mathrm{K}$ Art Hall should be proven. Towards this end, as shown in Figure 2, acoustic simulation modeling for $\mathrm{K}$ Art Hall was conducted using the CATT v8.0 program [33]. The acoustic simulation modeling process is as follows.

First, the two sound sources and 10 sound receivers in K Art Hall were determined. According to Korean Standard for the Measurement of the RT of Rooms with Reference to the other acoustical parameters (KS F 2864:2002) [34], if the number of indoor seats is 500 or below, over six sound receivers should be installed. As $\mathrm{K}$ Art Hall was designed to have 390 seats, it satisfies the criterion set by KS F 2864:2002.

Second, the sound absorption coefficients by frequency band for the finish materials in K Art Hall were determined (refer to Table 2). This is because the sound quality of an indoor music venue is dependent on the sound reflection and sound absorption coefficients of the finish materials based on the frequency bands of sounds. Thus, the sound absorption coefficients by frequency band for the finish materials were determined from the locations of the 10 sound receivers in K Art Hall, and acoustic simulation was conducted. 


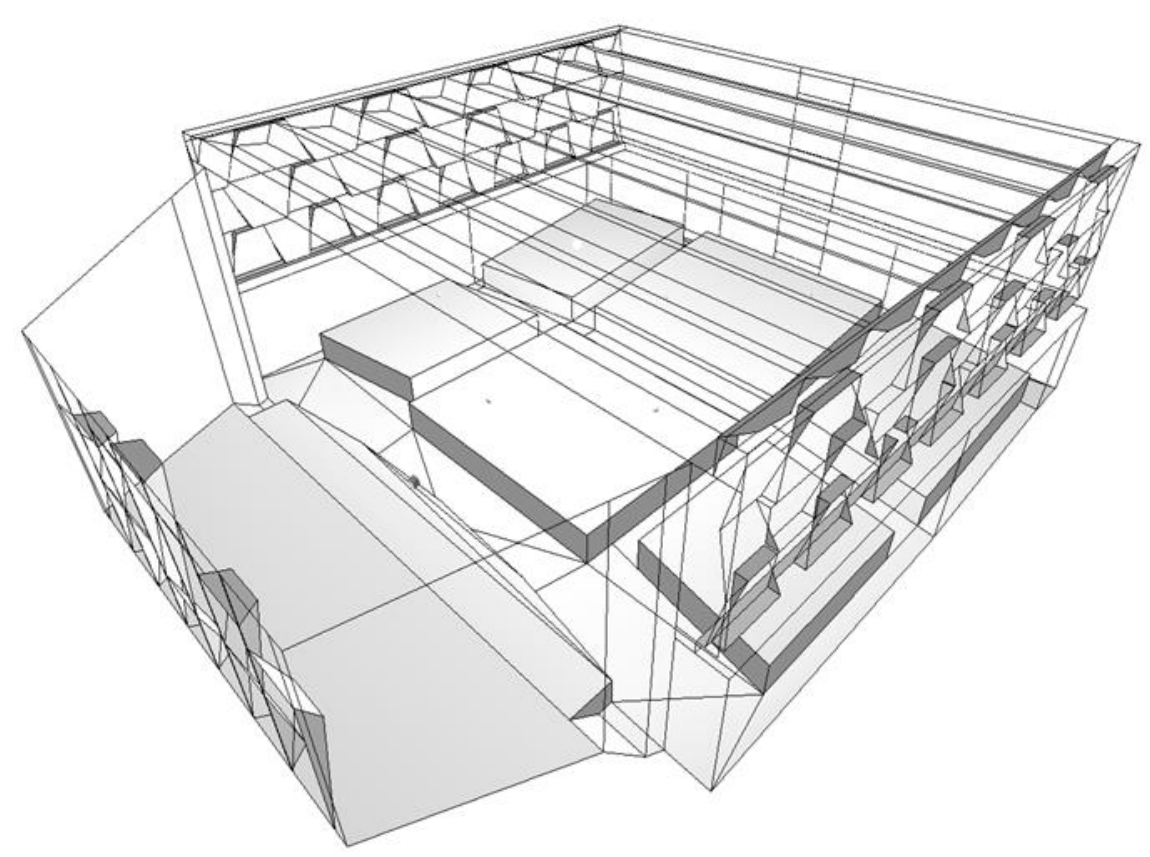

Figure 2. 3D acoustic simulation modeling of K Art Hall using the CATT v.8.0 program

Figure 3 shows the acoustic simulation results of the classical concert hall in terms of the RT, C80, and LF distributions. First, the average RT values at $500 \mathrm{~Hz}$ and $1 \mathrm{kHz}$ were estimated at 1.4 seconds, satisfying the target RT (1.4 1.7 seconds). Second, the average C80 values at $500 \mathrm{~Hz}$ and 1 $\mathrm{kHz}$ were estimated at 1.6 and $1.2 \mathrm{~dB}$, respectively, satisfying the target $\mathrm{C} 80([-2,+2] \mathrm{dB})$. Third, the average LF values at $500 \mathrm{~Hz}$ and $1 \mathrm{kHz}$ were estimated at $29 \%$, respectively, satisfying the target LF $(10 \sim 35 \%)$. As a result, the acoustic design, including the interior and finish material plan, of K Art Hall was shown to be capable of achieving the optimal acoustic performance of the classical concert hall. 


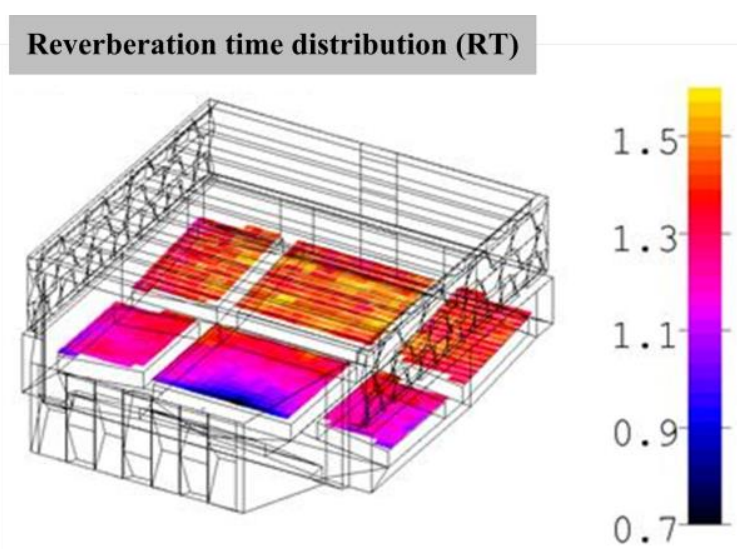

(A-1) Reverberation time (RT) distribution at $500 \mathrm{~Hz}$
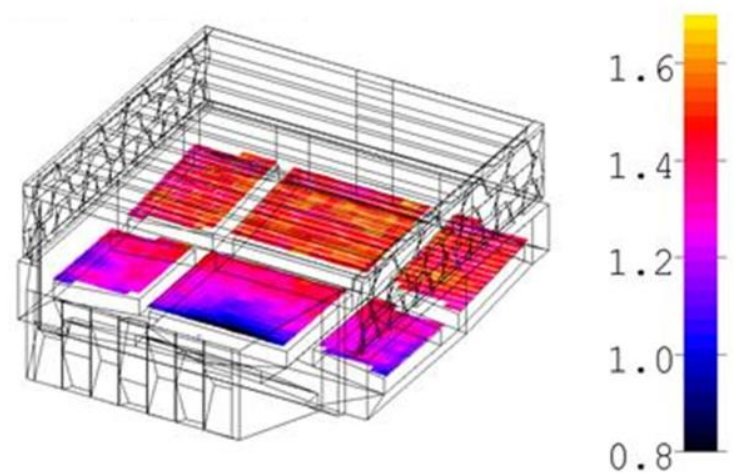

(A-2) Reverberation time (RT) distribution at $1 \mathrm{kHz}$

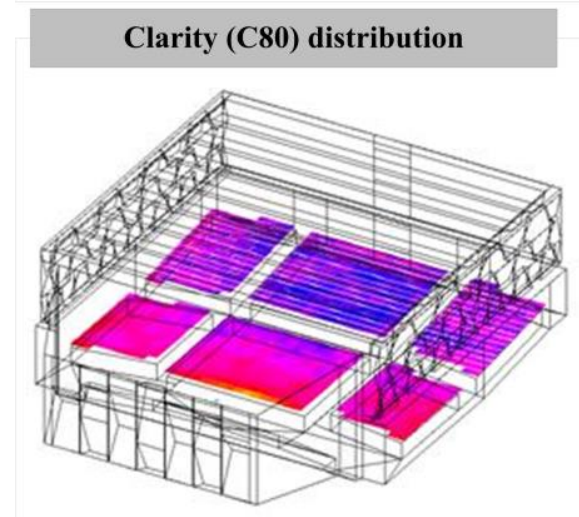

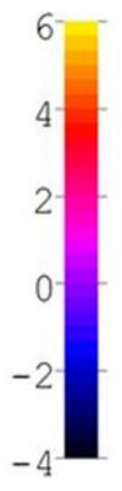

(B-1) Clarity (C80) distribution at $500 \mathrm{~Hz}$
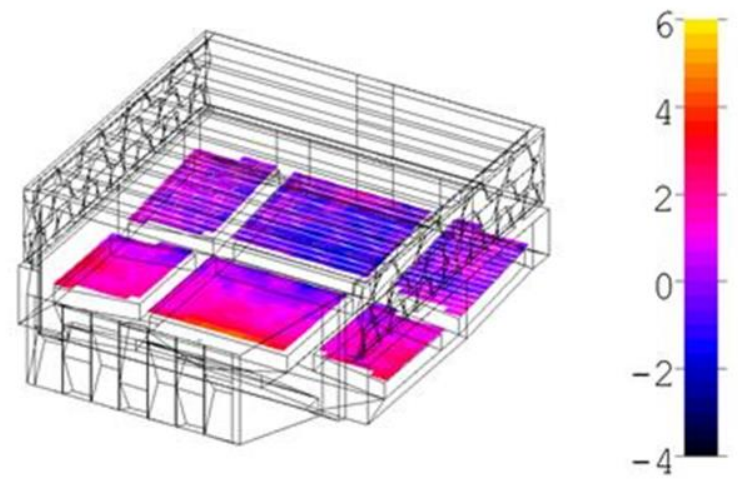

(B-2) Clarity (C80) distribution at $1 \mathrm{kHz}$

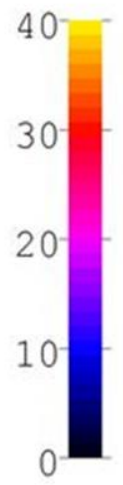

(C-1) Lateral fraction (LF) distribution at $500 \mathrm{~Hz}$

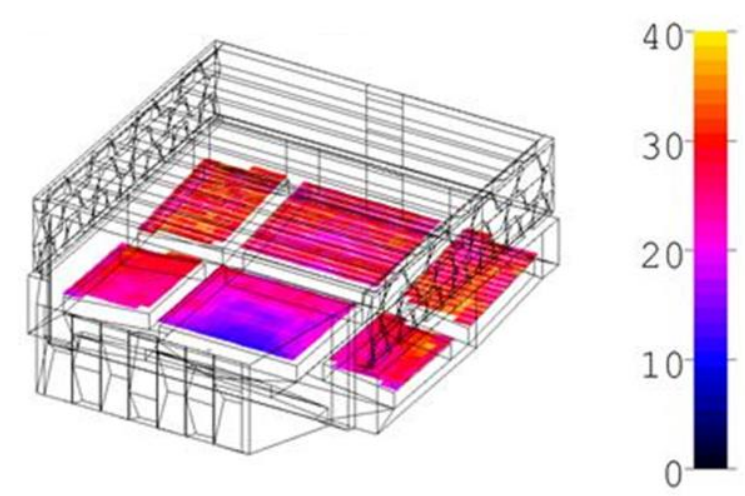

(C-2) Lateral fraction (LF) distribution at $1 \mathrm{kHz}$

Figure 3. Acoustic simulation results of the classical concert hall in terms of RT, C80, and LF distributions

\section{Evaluation of the acoustic performance through site measurements}

To verify if the acoustic design (refer to section 2) and acoustic simulation results (refer to section 3) of K Art Hall had been reflected in its construction, evaluation of the hall's acoustic performance through site measurements was conducted in terms of the RT, C80, and LF. As mentioned in section 3 (acoustic simulation modeling of the classical concert hall using an acoustic simulation program), the site measurement of the acoustic performance of $\mathrm{K}$ Art Hall was conducted based on KS F 2864:2002 [34].

First, the site measurement for evaluating the acoustic performance was conducted using various acoustic measuring equipment (refer to Table 3). The e-Sweep signal generated from the non- 
directional speaker in Dirac 3.0 is collected with a microphone, which is a sound receiver. This study performed a total of three site measurements (1st measurement: August 20, 2015; 2nd measurement: October 15, 2015; and 3rd measurement: December 8, 2015) for evaluating the acoustic performance of the concert hall, and analyzed the three acoustic parameters (i.e., RT, C80, and LF).

Second, the two sound sources (i.e., S-01 and S-02) and 10 sound receivers (i.e., P-01, P-02, P-03, P-04, P-05, P-06, P-07, P-08, P-09, and P-10) in K Art Hall were determined for conducting site measurement (refer to Figure 4). As K Art Hall was designed symmetrically, the site measurement for the acoustic performance of the left side from the center was performed. As mentioned in section 3.2 (analysis of the acoustic performance through acoustic simulation modeling), K Art Hall was designed to have 390 seats, thus satisfying the criterion proposed by KS F 2864:2002 (installing six or more sound receivers if the number of seats is 500 or less) [34].

Table 3. Acoustic measuring equipment for evaluating the acoustic performance of the K Art Hall

\begin{tabular}{ccc}
\hline Class & Equipment model & Manufacturer \\
\hline Audio analyzer & Dirac 3.0 (Dual Input Room Acoustic Calculator) & Acoustic Engineering \\
Microphone & C414 B-ULS (Bidirectional Microphone) & AKG \\
SPL meter & SVAN 943 Bz (Sound Analyzer) & SVANTEK \\
Amplifier & E45 (DBX 386 Vacuum Tube) & MC2 AUDIO \\
Speaker & Pyrite OS12 (Omni Directional Loudspeaker) & Acoustic Engineering \\
\hline
\end{tabular}

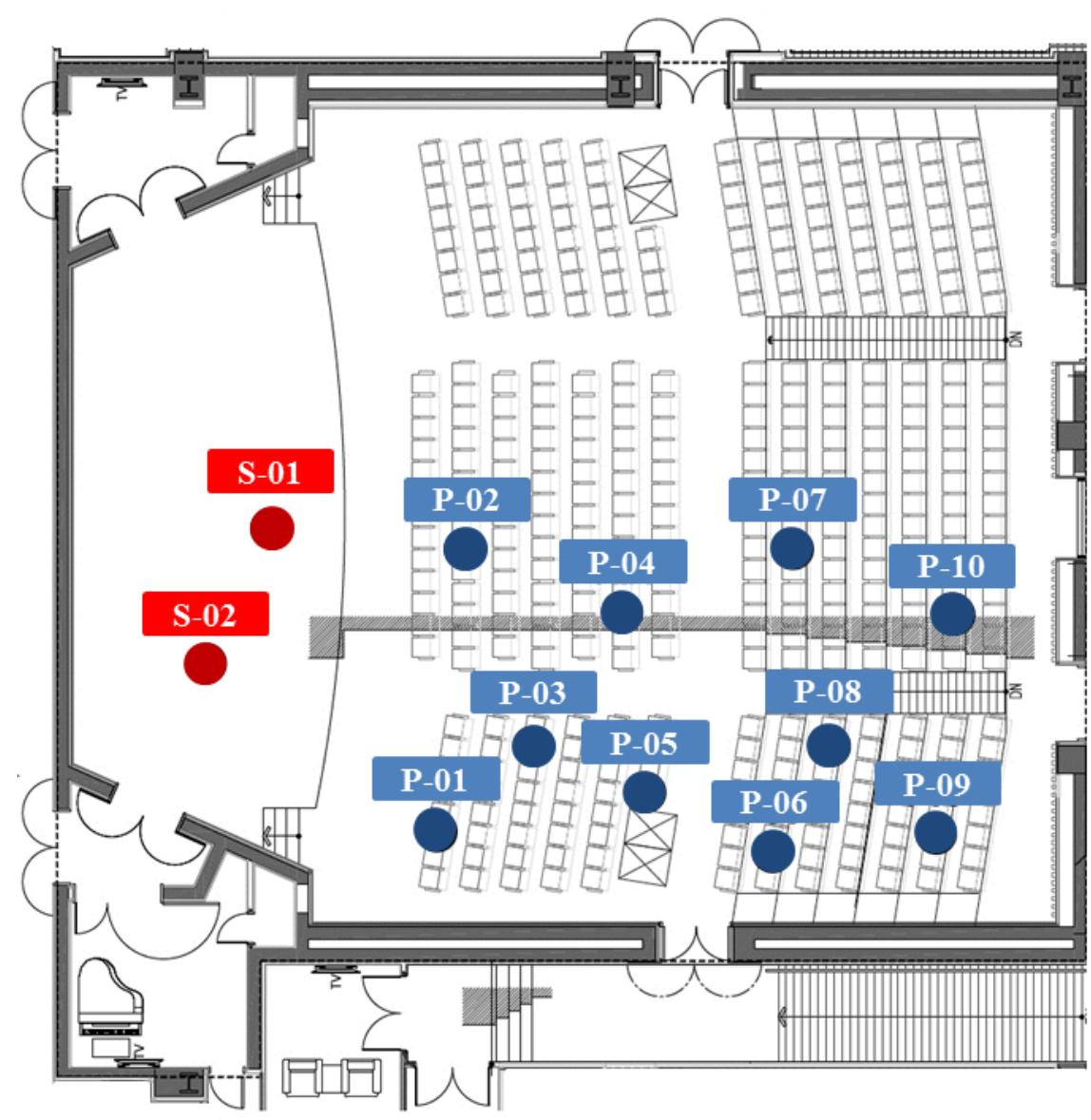

Figure 4. Locations of two sound sources and 10 sound receivers in the K Art Hall

Figure 5 shows the site measurement results of RT by frequency band. When the frequency bands were 125, 250, 500, 1,000, 2,000, and 4,000 Hz, the average RT values were $1.50 \mathrm{~s}$ (range: 1.46 $1.55 \mathrm{~s}$ ), $1.57 \mathrm{~s}$ (range: $1.54 \sim 1.61 \mathrm{~s}$ ), $1.55 \mathrm{~s}$ (range: $1.50 \sim 1.60 \mathrm{~s}$ ), $1.59 \mathrm{~s}$ (range: $1.55 \sim 1.60 \mathrm{~s}$ ), $1.64 \mathrm{~s}$ (range: $1.62 \sim 1.65 \mathrm{~s}$ ), and $1.51 \mathrm{~s}$ (range: $1.44 \sim 1.57 \mathrm{~s}$ ). These measured RT values were shown to satisfy the 
target RT value (1.4 1.7 seconds) requested by the classical concert hall. Meanwhile, the standard deviation (SD) of the RT based on the frequency band and location of the sound receiver was shown to be 0.063 (SD of the 1st measurement: 0.048, SD of the 2nd measurement: 0.066 , and SD of the 3rd measurement: 0.074). Due to this small deviation, equally distributed sound in all the seats can result when sounds are delivered from the stage to the auditorium. Also, compared to the RT value of the mid-frequency band (500 and 1,000 Hz), that of the low-frequency band $(125$ and $250 \mathrm{~Hz})$ or of the high-frequency band $(2,000$ and $4,000 \mathrm{~Hz})$ is shorter, and therefore, suitable low-pitched sounds can be acquired even when the auditorium is filled to capacity.

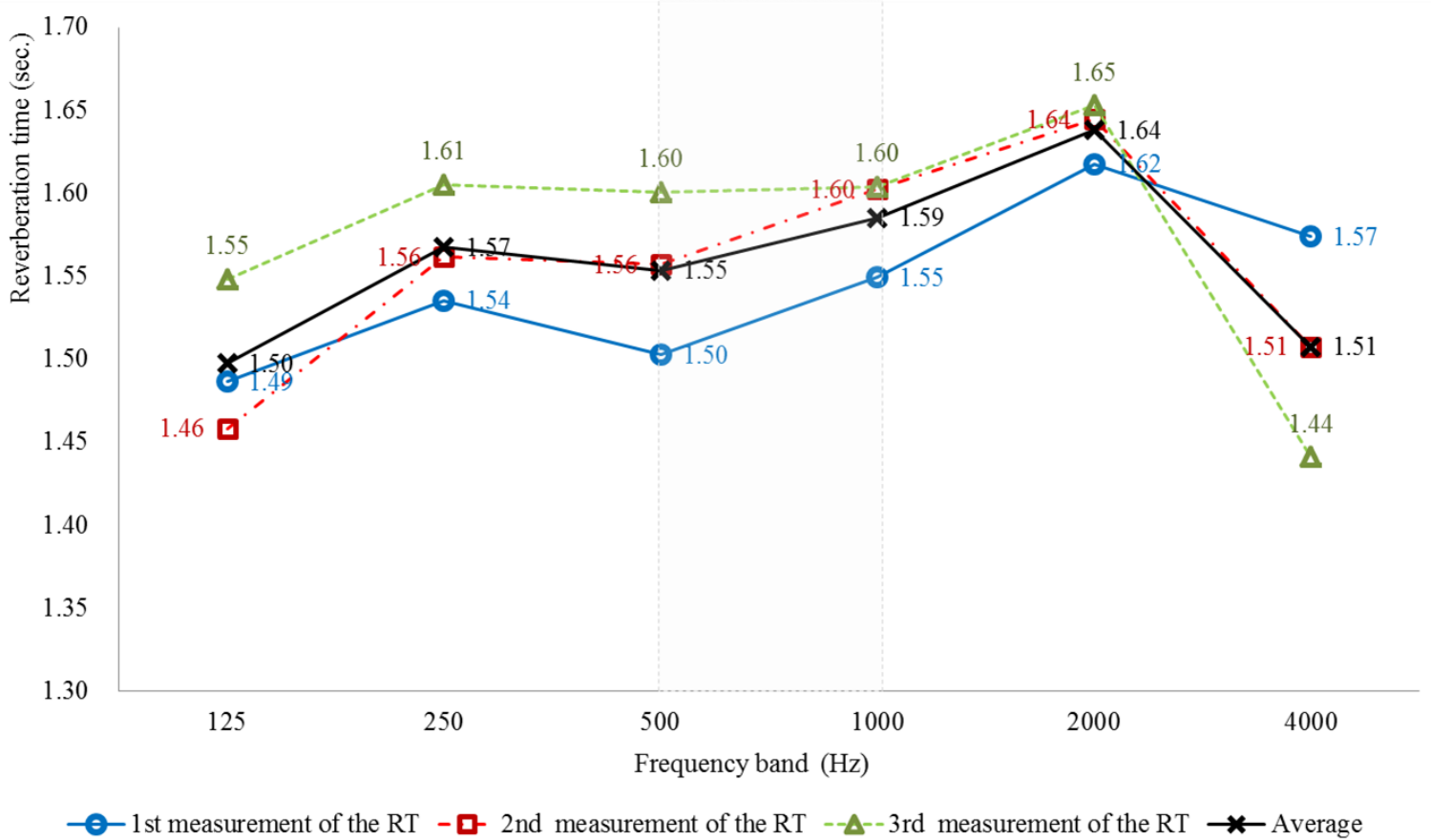

Figure 5. Site measurement results of the RT by frequency band

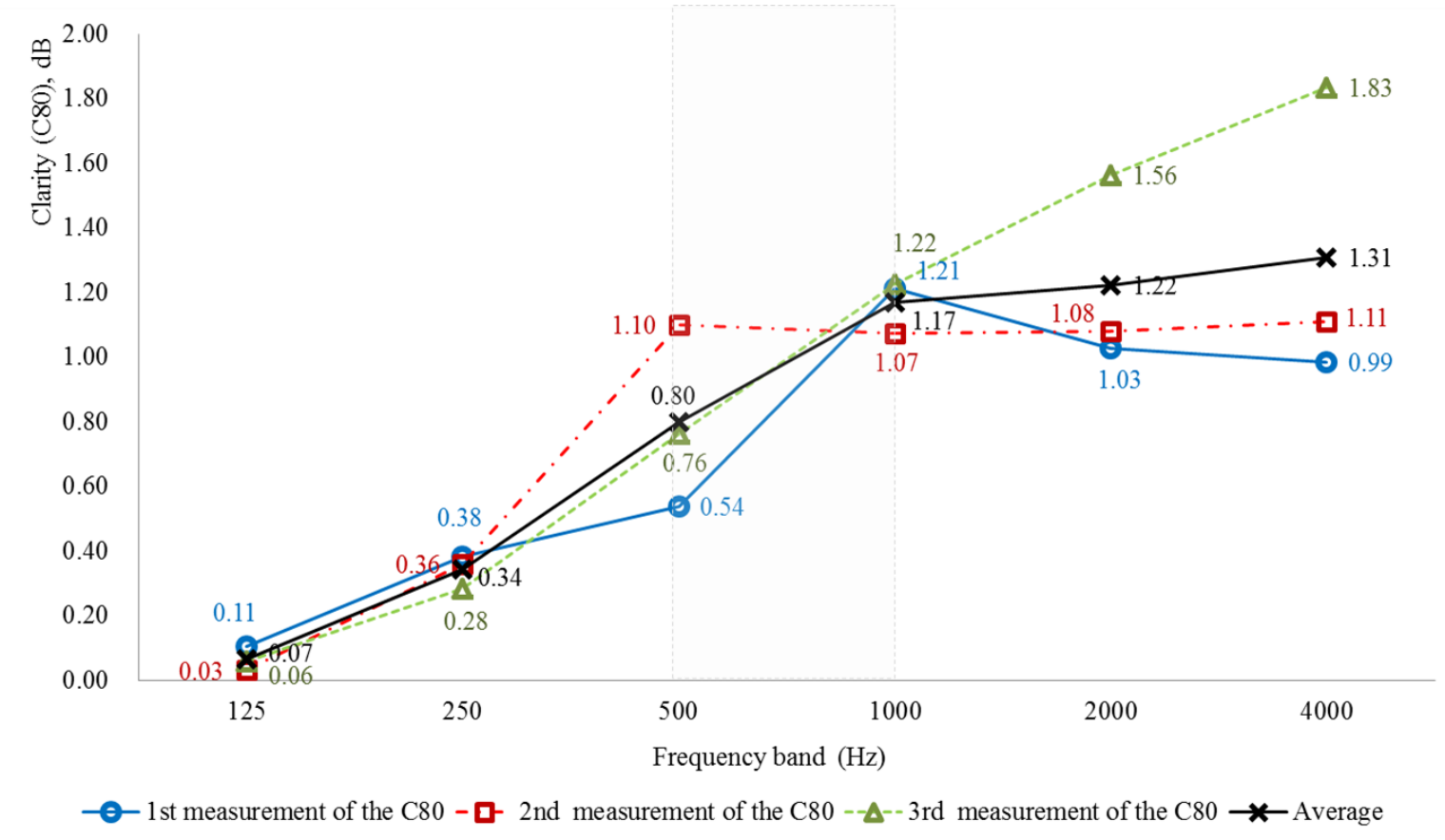

Figure 6. Site measurement results of the $\mathrm{C} 80$ by frequency band 
Figure 6 shows the site measurement results of $\mathrm{C} 80$ by frequency band. When the frequency bands were 125, 250, 500, 1,000, 2,000, and 4,000 Hz, the average C80 values were $0.07 \mathrm{~dB}$ (range: 0.03 $\sim 0.11 \mathrm{~dB}$ ), $0.34 \mathrm{~dB}$ (range: $0.28 \sim 0.38 \mathrm{~dB}$ ), $0.80 \mathrm{~dB}$ (range: $0.54 \sim 1.10 \mathrm{~dB}$ ), $1.17 \mathrm{~dB}$ (range: $1.07 \sim 1.22$ $\mathrm{dB}$ ), $1.22 \mathrm{~dB}$ (range: $1.03 \sim 1.56 \mathrm{~dB}$ ), and $1.31 \mathrm{~dB}$ (range: $0.99 \sim 1.83 \mathrm{~dB}$ ). These measured $\mathrm{C} 80$ values were shown to satisfy the target $C 80$ value $([-2,+2] \mathrm{dB})$ required by the classical concert hall. As a result, the average $\mathrm{C} 80$ value in the mid-frequency band $(500$ and $1,000 \mathrm{~Hz})$ was $0.96 \mathrm{~dB}$, resulting in superior clarity with somewhat faster music.

Figure 7 shows the site measurement results of LF by frequency band. When the frequency bands were $125,250,500,1,000,2,000$, and $4,000 \mathrm{~Hz}$, the average $\mathrm{LF}$ values were $24.15 \%$ (range: 19.30 $\sim 31.90 \%$ ), 22.22\% (range: $21.05 \sim 24.10 \%$ ), 27.33\% (range: $25.15 \sim 29.95 \%$ ), 29.22\% (range: $27.15 \sim$ $33.35 \%$ ), $26.12 \%$ (range: $24.15 \sim 27.85 \%$ ), and 31.37\% (range: $28.90 \sim 33.55 \%$ ). These measured LF values were shown to satisfy the target LF value $(10 \sim 30 \%)$ required by the classical concert hall. As a result, the average LF value in the mid-frequency band ( 500 and $1,000 \mathrm{~Hz}$ ) is $28 \%$, and thanks to the sufficient sound refection by the sidewalls, the audience in the auditorium can experience a satisfactory sense of spatiality.

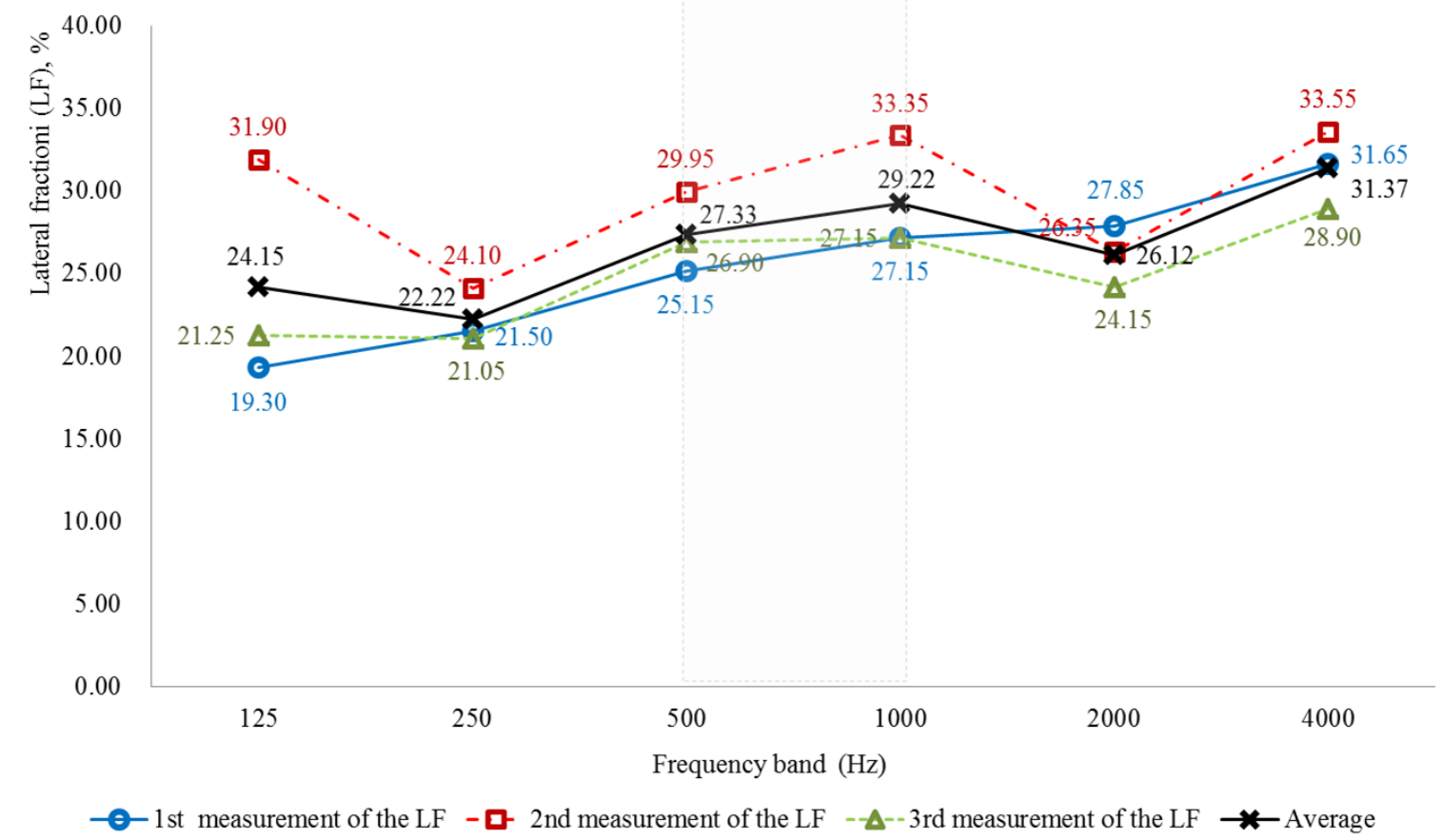

Figure 7. Site measurement results of the LF by frequency band

\section{Conclusion}

Due to the increasing demand for the classical music, there is an increasing demand for the acoustic performance as well as a quantitative increase in the classic concert hall. However, unlike the large-scale concert hall, the small and medium-scale concert hall limits the systematic acoustic design because it performs the acoustic planning after the architectural planning phase is completed. In particular, there are only a small number of studies that have been carried out on acoustic design in the early planning phase and the acoustic evaluation in the operation phase on small and mediumscale concert halls. Thus, this study intends to carry out an acoustic design of the classical concert hall and evaluation of its acoustic performance on a small and medium-scale classical concert hall (K Art Hall located in Seoul, South Korea).

The analysis results are as follows. First, the shapes of the room, and finish materials as an interior acoustic design were determined based on literature reviews. The shape of the room was determined to be a 'Shoe-Box' type suitable for small and medium-scale classical concert halls, and the finish materials were determined by considering the sound reflation and sound diffusion for the stage, auditorium, and so on. Second, the average values of the RT, C80, and LF using the acoustic 
simulation modeling were estimated at 1.4 second, $1.2 \sim 1.6 \mathrm{~dB}$, and $29 \%$, respectively. Third, the average values of the RT, C80, and LF through site measuring were measured at $1.5 \sim 1.64$ second, $0.07 \sim 1.31 \mathrm{~dB}$, and $22.22 \sim 31.37 \%$, respectively. As a result, the analysis results of the acoustic simulation modeling and site measuring based on the acoustic design were all satisfied the target acoustic performance (target RT: within 14 seconds, target C 80 : $-2 \mathrm{~dB}$ or more $+2 \mathrm{~dB}$ or less, and target LF: $10 \sim 35 \%$ ). The results of this study will help decision-makers (i.e., owners, and construction managers.) to plan classical concert halls in terms of the RT, C80, and LF.

Acknowledgements: This work was supported by a grant (17CTAP-C114950-02) from Technology Advancement Research Program (TARP) funded by Ministry of Land, Infrastructure and Transport of Korean government.

Author Contributions: Kwangbok Jeong, Taehoon Hong, Sung Hoon Kim, Jimin Kim, and Sangyoub Lee wrote the paper.

Conflicts of Interest: The authors declare no conflict of interest.

\section{Abbreviations}

C80: Clarity

CATT: Computer Aided Theater Technique

LF: Lateral fraction

RT: Reverberation time

SD: Standard deviation

\section{References}

1. Barron, M. Subjective study of Bitish symphony concert halls. Acustica 1988, 66, 1-14.

2. Beranek, L.L. Music, acoustics and architecture. John Wiley \& Sons, New York, U.S, 1962.

3. San, M.R.; Arana M. Predicted and experimental results of acoustic parameters in the new Symphony Hall in Pamplona, Spain. Applied Acoustics 2006, 67, 1-14, doi: 10.1016/j.apacoust.2005.04.011.

4. Galindo, M.; Zamarreño, T.; Girón, S. Acoustic simulations of Mudejar-Gothic churches. The Journal of the Acoustical Society of America 2009, 126, 1207-1218, doi: 10.1121/1.3180632.

5. Galiana, M.; Llinares, C.; Page, A. Impact of architectural variables on acoustic perception in concert halls. Journal of Environmental Psychology 2016, 48, 108-119, doi: 10.1016/j.jenvp.2016.09.003.

6. Ramakrishnan, R.; Dumoulin, R. Acoustics of a music venue/bar - a case study. Buildings 2016, 6(1), 11, doi: 10.3390/buildings6010011.

7. Barron, M.; Kissner, S. A possible acoustic design approach for multi-purpose auditoria suitable for both speech and music. Applied Acoustics 2017, 115, 42-49, doi: 10.1016/j.apacoust.2016.08.018.

8. Garrido, J.A.; Zamarreño, T.; Girón, S. Virtual models for the prediction of acoustic fields of Manuel de Falla Auditorium in Granada, Spain. Applied Acoustics 2012, 73, 921-935, doi: 10.1016/j.apacoust.2012.03.015.

9. Navarro, J.M.; Escolano, J. Simulation of building indoor acoustics using an acoustic diffusion equation model. Journal of Building Performance Simulation 2015, 8(2), 3-14, doi: 10.1080/19401493.2013.850534.

10. Martins, C.; Santos, P.; Almeida, P.; Godlinho, L.; Dias, A. Acoustic performance of timber and timberconcrete floors. Construction and Building Materials 2015, 101, 684-691, doi: 10.1016/j.conbuildmat.2015.10.142.

11. De Sant'Ana, D.Q.; Zannin, P.H.T. Acoustic evaluation of a contemporary church based on in situ measurements of reverberation time, definition, and computer-predicted speech transmission index. Building and Environment 2011, 46, 511-517, doi: 10.1016/j.buildenv.2010.08.015.

12. Arets, M.; Orlowski, R. Sound strength and reverberation time in small concert halls. Applied Acoustics 2009, 70, 1099-1110, doi: 10.1016/j.apacoust.2009.02.001.

13. Mak, C.M.; Wang, Z. Recent advances in building acoustics: An overview of prediction methods and their applications. Building and Environment 2015, 91, 118-126, doi: 10.1016/j.buildenv.2015.03.017. 
14. Pätynen, J.; Tervo, S.; Robinson, P.W.; Lokki, T. Concert halls with strong lateral reflections enhance musical dynamics. Proceedings of the National Academy of Sciences of the United States of America 2014,111(12), 4409-4414, doi: 10.1073/pnas.1319976111.

15. Beranek, L. Concert hall acoustics. Architectural Science Review 2011, 54, 5-14, doi: doi:10.3763/asre.2010.0059.

16. Lokki, T. Why is so hard to design a concert hall with excellent acoustics?. Proceedings of Acoustics 2016, Brisbane, Australia, 9-11 November 2016.

17. Haan, C.; Fricke, F.R. An Evaluation of the Importance of Surface Diffusivity in Concert Halls. Applied Acoustics 1997, 51(1), 53-69, doi: 10.1016/S0003-682X(96)00060-6.

18. Husin, H.N.; Mustapa, S.A.H.B.S.; Kamal, K.S. Acoustical requirements of Dewan Filharmonik Petronas, Malaysia. Proceedings of the $38^{\text {th }}$ International Conference of Architectural Science Association NZAScA, Launceston, Tasmania, 10-12 November 2004.

19. Gul, Z.S.; Caliskan, M. Acoustical design of Turkish Religious Affairs Mosque. Proceedings of Meetings on Acoustics, Montreal, Canada, 2-7 June 2013.

20. Sato, S.; Kim, Y.H.; Lee, H.M.; Jeon, J.Y. Objective evaluations of sound scattering by using scale models and computer simulation. Proceedings of the $19^{\text {th }}$ International Congress on Acoustics Madrid, Madrid, Spain, 2-7 September 2007.

21. Kitamura, K.; Fukunishi, T.; Furukawa, S. A study of design methods for the variable reverberation units in auditoria. Preceding the $18^{\text {th }}$ International Congress on Acoustics, Kyoto, Japan, 4-9 April 2004.

22. Komoda, M.; Hakozaki, A.; Toyota, Y. Acoustical Design of New Danish Radio Concert Hall. Proceedings of the International Symposium on Room Acoustics, Melbourne, Australia, 29-31 August 2010.

23. Eyring, C.F. Reverberation time measurements in coupled rooms. The Journal of the Acoustical Society of America 1931, 3, 181-206, doi: 10.1121/1.1915555.

24. Barron, M. Auditorium acoustics and architectural design. 1st ed. Taylor \& Francis, London \& New York, U.K \& U.S, 1993.

25. Egan, D.M., Architectural acoustics. McGraw-Hill, Inc. p.64, 1988.

26. Kim, J. Architectural acoustics design. Sejin Co., Republic of Korea, 2004, pp.361.

27. Cox, T.J.; Davies, W.J.; Lam, Y.W. The sensitivity of listeners to early sound field changes in auditoria. Acustica 1993, 79, 27-41.

28. Bradley, J.S.; Reich, R.; Norcross, S.G. A just noticeable difference in C50 for speech. Applied Acoustics 1999, 58 99-108, doi: 10.1016/S0003-682X(98)00075-9.

29. Barron M. Objective assessment of concert hall acoustics using temporal energy analysis. Applied Acoustics 2013, 74(7), 936-944, doi: 10.1016/j.apacoust.2013.01.006.

30. Barron, M. Using the standard on objective measures for concert auditoria, ISO 3382, to give reliable results. Acoustical Science and Technology 2005, 26(2), 162-169, doi: 10.1250/ast.26.162.

31. Schuitman, J.V.D; De Vries, D.; Lindau, A. Deriving content-specific measures of room acoustic perception using a binaural, nonlinear auditory model. Journal of the Acoustical Society of America 2013, 133(3), 1572-1585, doi: 10.1121/1.4789357.

32. Fasold. W.; Veres. E. Schallschutz und raumakustik in der praxis. Planungsbeispiele und konstruktive lösungen. 1st ed. Berlin: Verlag für bauwesen; 1998.

33. Dalenbäck, B.I. Room acoustic prediction based on a unified treatment of diffuse and specular reflection. Journal of the Acoustical Society of America 1996, 100(2), 899-909, doi: doi.org/10.1121/1.416249.

34. Korean Standard for the Measurement of the reverberation time of rooms with reference to the other acoustical parameters. Available online: http://www.kssn.net/StdKS/KS_detail.asp?K1=F\&K2=2864\&K3=3 (accessed on 25th October 2017). 